\title{
ARTICLE OPEN \\ Creating multiferroic and conductive domain walls in common ferroelastic compounds
}

\author{
Hong Jian Zhao $\mathbb{D}^{1,3}$ and Jorge Íñiguez $\mathbb{D}^{1,2}$
}

Domain walls in ferroelectrics and ferroelastics often present peculiar functional properties, offering an intriguing route toward the design of nano-devices. Here we use first-principles simulations to illustrate an approach for engineering such walls, working with representative ferroelastic perovskites $\mathrm{LaGaO}_{3}$ and $\mathrm{CaTiO}_{3}$ (insulating, non-magnetic, non-polar). We show that a wide range of substitutional dopants can be used to create long-range-ordered structures confined within the walls of these compounds, yielding functional interfaces with tailor-made properties. We thus identify clear-cut strategies to produce metallic walls within an insulating matrix. Further, we find ways to create magnetic walls that also display ferroelectric order (proper or improper), thus providing an original route to obtain magnetoelectric multiferroics. Given the recent developments on the preparation of high-density domain structures in perovskite films, our results suggest a definite path toward new functional nano-materials.

npj Computational Materials (2019)5:92; https://doi.org/10.1038/s41524-019-0229-5

\section{INTRODUCTION}

In the search for new functional nano-materials, the structural domain walls (DWs) of ferroelectric and ferroelastic compounds stand out as an intriguing possibility. ${ }^{1-4}$ These DWs can be prepared in very dense and well-ordered arrays-e.g., by growing thin films on appropriate substrates-so as to occupy a significant volume fraction of the sample. Further, they can be manipulated in increasingly precise and flexible ways, ${ }^{5-7}$ raising hopes of a potential dynamical control. Finally, their properties often differ from those of the domains themselves as, e.g., they are typically observed to be much more conductive. ${ }^{8-11}$ Hence, structural DWs may open the door to the design of novel, actable, functional nano-materials. This engineering strategy is best captured by the motto "the wall is the device", and is a major focus of attention for the community working on ferroics, particularly $\mathrm{ABO}_{3}$ perovskite oxides and related compounds.

The unique DW properties mostly derive from their peculiar structure. Domains in stable low-energy configurations meet (clash) at the DWS, forcing the DW atoms to adopt unusual highenergy arrangements that would not occur otherwise. Hence, it is no surprise the properties (structural, electronic, magnetic, optical) differ from those of the domains. Also, DWs can be expected to be the preferred location of all sort of point defects, chiefly oxygen vacancies in perovskite oxides, which probably explains most of the observed cases of enhanced conductivity. ${ }^{10-12}$ In fact, DWs can even host ordered arrays of defects, as, e.g., was found in $\mathrm{TbMnO}_{3}$ thin films: Farokhipoor et al. ${ }^{13}$ observed that the walls in their samples have a distinct stoichiometry, with half of the Tb cations substituted by $\mathrm{Mn}$ 's and forming a unique functional interface-arguably, a genuine two-dimensional (2D) crystal-for which no analogue seems to exist in bulk form. Further, this chemical substitution gives the $\mathrm{TbMnO}_{3}$ walls peculiar magnetic properties, which were characterized experimentally and explained theoretically in the cited work. Hence, according to ref. ${ }^{13}$ and other experimental works, ${ }^{14,15}$ not only can we expect different behaviors at structural DWs, but we can also attempt to create completely new nano-materials within them.

Here we use first-principles methods based on density functional theory (DFT) to explore the use of chemical doping to create functional interfaces at the domain walls of common ferroelastic materials. Our results provide ample support for this materials-design strategy, predicting the spontaneous occurrence of long-range-ordered substitutions for a large variety of dopants. Further, we identify specific strategies to obtain walls with allimportant properties, including conductivity and magnetoelectric multiferroism.

\section{RESULTS}

Preferential doping at domain walls

We focus our attention on the most common phase in the perovskite family, namely, the orthorhombic structure characterized by a combination of antiphase (about the pseudo-cubic [110] axis) and in-phase (about [001]) rotations of the $\mathrm{O}_{6}$ oxygen octahedra (see Fig. 1). This phase has space group Pbnm and is characterized as " $a-a^{-} c^{+"}$ in Glazer's self-explanatory notation. ${ }^{16}$ This is the phase of $\mathrm{TbMnO}_{3}$, the material investigated in ref. ${ }^{13}$, and is also the structure displayed by about half of all perovskite oxides at ambient conditions. ${ }^{17,18}$ Here we consider representative compounds (mostly $\mathrm{LaGaO}_{3}$, as well as $\mathrm{CaTiO}_{3}$ and $\mathrm{TbMnO}_{3}$ for additional tests) that are well known and widely available, to emphasize the generality and experimental feasibility of our predictions.

Thin films of Pbnm perovskites tend to present ferroelastic domains that help accommodate the strain imposed by the substrate. In Fig. 1, we show an example of the type of ferroelastic

\footnotetext{
${ }^{1}$ Materials Research and Technology Department, Luxembourg Institute of Science and Technology (LIST), Avenue des Hauts-Fourneaux 5, L-4362 Esch/Alzette, Luxembourg;

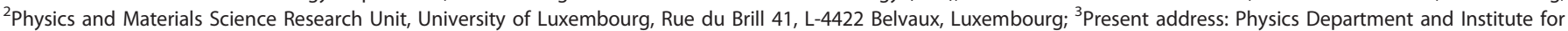
Nanoscience and Engineering, University of Arkansas, Fayetteville, AR 72701, USA

Correspondence: Hong Jian Zhao (hz001@uark.edu) or Jorge Îñiguez (jorge.iniguez@list.lu)
}

Received: 18 March 2019 Accepted: 2 August 2019

Published online: 12 September 2019 

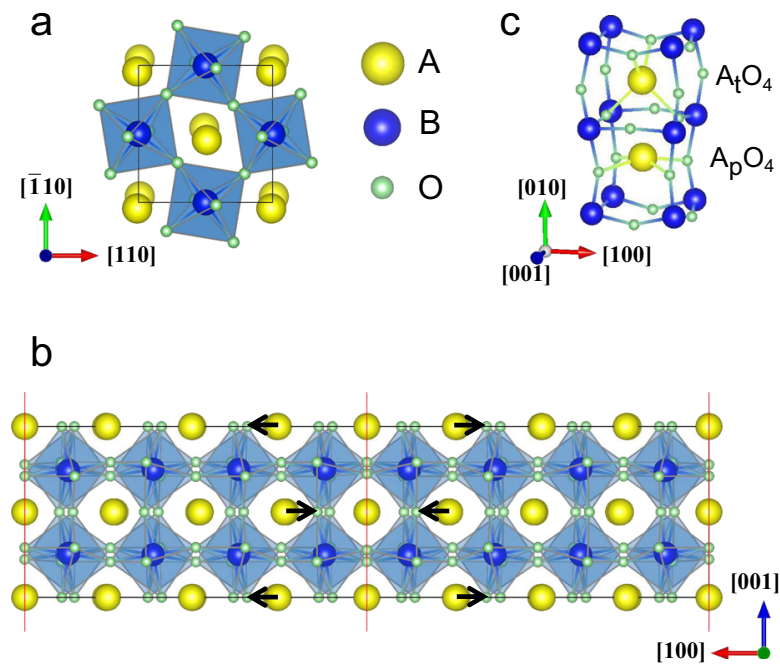

Fig. 1 Structural details. a Orthorhombic phase that is typical of most perovskite oxides, in particular the compounds investigated here $\left(\mathrm{LaGaO}_{3}, \mathrm{CaTiO}_{3}\right.$, and $\left.\mathrm{TbMnO}_{3}\right)$. This structure is characterized by tilts of the $\mathrm{O}_{6}$ octahedra (in a pattern denoted as $a^{-} a^{-} c^{+}$in Glazer's notation ${ }^{16}$ ) and anti-polar motions of the $A$ cations. $\mathbf{b}$ Sketch of the 160-atom periodically repeated supercell considered in this work for most multi-domain simulations. Red lines mark the DW positions. Black arrows emphasize how the $A$ cations inside the domains move toward (away from) the central wall, creating compressed (expanded) $A$ sites at it. c Structure of the compressed $A$ sites at the wall. There are two distinct situations, with a characteristic square-planar $\left(A_{p}\right)$ and tetrahedral $\left(A_{t}\right)$ oxygen environment of the $A$ cations, respectively. All axes are referred to the pseudo-cubic setting associated to the elemental 5-atom perovskite cell

walls typical of such multi-domain states; more specifically, the walls sketched in the figure are essentially those characterized experimentally in $\mathrm{TbMnO}_{3}{ }^{13}$ and will be our focus of attention here. Further, as emphasized in Fig. 1, the Pbnm phase presents a pattern of A-cation displacements yielding compressed and expanded sites at its ferroelastic wall. The authors of ref. ${ }^{13}$ proposed that such steric constraints are key to the observed long-range-ordered chemical substitutions at the walls of $\mathrm{TbMnO}_{3}$, postulating that it is energetically favorable for relatively small cations $\left(\mathrm{Mn}^{3+}\right.$ in their case) to occupy the compressed $A_{\mathrm{p}}$ and $A_{\mathrm{t}}$ sites. Here we corroborate the correctness of such an interpretation, and its relevance to other cases.

We work with the structural model in Fig. $1 \mathrm{~b}$ of a DW in $\mathrm{LaGaO}_{3}$ and $\mathrm{CaTiO}_{3}$, and compute the energy of various substitutional defects (small cations belonging to the first row of $3 d$ transition metals, as well as a few $4 d$ and $5 d$ elements) at $A$ sites located at and around the DW. (See Methods and Supplementary Fig. 1 for details of the simulations.) Typically, for $3 d$ dopants replacing La, we find that substitution at the compressed DW site is favored by 0.2-0.6 eV as compared with a substitution inside the domain, and by at least $0.1 \mathrm{eV}$ as compared with a substitution at an expanded DW site. (In a few cases we find that substitution within the domain is competitive with substitution at the expanded DW site. We also consider substitution for $\mathrm{Ga}$, and obtain no significant difference between domains and walls.) In addition, we find no clear trend regarding the occupation of the tetrahedral $\left(A_{\mathrm{t}}\right)$ vs square-planar $\left(A_{\mathrm{p}}\right)$ compressed sites; preference for one or the other depends on the particular $3 d$ dopant and involves relatively small energy differences. Our calculations for $4 d$ and $5 d$ elements yield the same general conclusions; yet, we find that energy differences can be larger (up to $1 \mathrm{eV}$ ) in those cases, which can be traced back to the fact that the bigger $4 d$ and $5 d$ cations sometimes adopt particularly stable low-spin states at the compressed DW sites. (With the exception of some cases involving $\mathrm{Co}$ and $\mathrm{Ni}$, for the $3 d$ dopants we always obtain high-spin states from our simulations; see Supplementary Tables 1 and 2 for details).

We must note that, for all dopants considered, it was all but impossible to obtain a systematic collection of defect formation energies, e.g., as a function of distance to the DW and $A$ site at which the substitution occurs. The difficulty pertains to the simulation of defects within the domains or at expanded DW sites: Frequently, our simulated annealing and ensuing structural relaxation results in some sort of atomic rearrangement (e.g., a shift of the DW plane) so that the dopant ends up located at a compressed site. This somewhat surprising outcome further emphasizes the preference of the dopants to occupy the $A_{\mathrm{p}}$ and $A_{\mathrm{t}}$ compressed positions at the DWs. In Supplementary Tables 3 and 4 we include details of our calculations for $\mathrm{Ti}$ and Fe dopants in $\mathrm{LaGaO}_{3}$, respectively, which are representative of most of the cases here considered. We also include our results for $\mathrm{Cr}$ and $\mathrm{Re}$ (Supplementary Tables 5 and 6, respectively), which feature some of the most spectacular dopant-induced structural rearrangements observed. (Cr's case is unique: it always forms a strongly distorted structure whose energy is largely independent from the position of the dopant at either wall or domain.) In addition, Supplementary Table 7 shows the case of $\mathrm{V}$ dopants in $\mathrm{CaTiO}_{3}$, which are representative of our results for this compound and corroborate our conclusions from the $\mathrm{LaGaO}_{3}$ case. (Because $\mathrm{CaTiO}_{3}$ displays a more robust structure of $\mathrm{O}_{6}$ tilts, treating this material is comparatively easy.) Finally, our calculations for $\mathrm{TbMnO}_{3}$ (not shown here) corroborate the preferential substitution at the compressive site (by about $1 \mathrm{eV}$ per dopant) observed experimentally in ref. ${ }^{13}$.

Hence, a first conclusion can be reached: Doping Pbnm perovskites with relative small transition-metal atoms is a viable strategy to create functional interfaces at their ferroelastic domain walls. Indeed, our stability calculations suggest that the investigated materials will try to accommodate the dopants by forming long-range-ordered substitutions at the compressed $A$ sites of their ferroelastic walls, exactly as observed in $\mathrm{TbMnO}_{3} \cdot{ }^{13}$ Related $2 \mathrm{D}$ defect structures have been reported in other materials, ${ }^{14,15}$ further supporting the experimental feasibility of the DWengineering concept here proposed.

To conclude this part, let us note that the above results suggest that our DWs will be pinned by the dopants, and we do not expect them to be easily mobile. Hence, any envisioned application should rely on the functional properties of static walls or groups of walls, in principle excluding the possibility of a dynamical reconfiguration of the multi-domain structure. Note, nevertheless, that our energy calculations do not permit a conclusive discussion of the kinetics of DW motion; for that, one would need a quantification of the associated energy barriers. A realistic calculation of such activation energies would require very large unit cells and costly transition-path optimizations, and is beyond the scope of the present work.

\section{Functional properties of doped walls}

Let us now discuss the physical properties of the chemically modified DWs with dopants at the compressed $A$ sites. We focus on the case of $\mathrm{LaGaO}_{3}$, an ideal material to host active DWs as it is particularly inert by itself. (Structurally, $\mathrm{LaGaO}_{3}$ presents the orthorhombic $a^{-} a^{-} c^{+}$phase in a wide range of temperatures and pressures, ${ }^{19,20}$ and has no tendency toward ferroelectricity; electronically, $\mathrm{LaGaO}_{3}$ is a non-magnetic insulator with a wide calculated gap of about $3.9 \mathrm{eV}$.)

Let us begin by noting that, by itself, the ferroelastic wall here considered has a negligible impact on the electronic structure of the pure compound. This can be easily appreciated by comparing the calculated electronic density of states of pure $\mathrm{LaGaO}_{3}$ in 


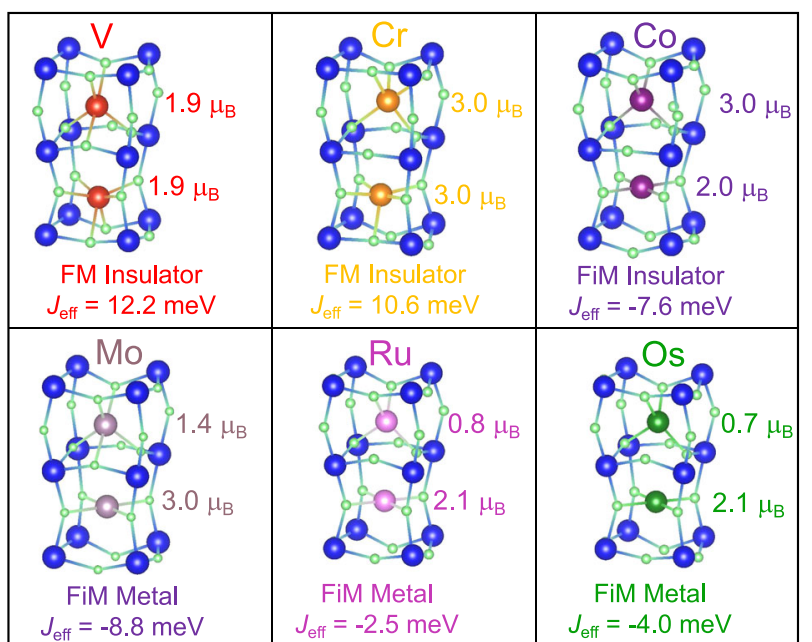

Fig. 2 Summary of results. Representative results obtained for DWs of $\mathrm{LaGaO}_{3}$ doped with the indicated transition-metal atoms. We show the relaxed structures of the compressed DW sites $A_{\mathrm{t}}$ and $A_{\mathrm{p}}$. We specify the magnetic moment of the dopant as quantified by a projection onto atomic-like orbitals (see Methods), the lowestenergy magnetic order, and the effective exchange interaction between nearest-neighboring spins. Note that for $\mathrm{Co}, \mathrm{Mo}, \mathrm{Ru}$, and Os, the observed lowest-energy anti-parallel spin alignment yields a ferrimagnetic (FiM) order, as the $A_{\mathrm{t}}$ and $A_{\mathrm{p}}$ sites display different magnetic moments

mono-domain and multi-domain configurations (see Supplementary Fig. 1). Hence, the electronic effects discussed below can be attributed to the dopants, not to the presence of a wall.

Figure 2 shows a selection of our results for $\mathrm{LaGaO}_{3}$. Let us begin by noting that we find many insulating solutions, but we also get some metallic and half-metallic configurations. Figure 3 shows the computed electronic density of states for representative cases, namely, V-doped (insulating), Mo-doped (half-metallic), and Os-doped (metallic). Generally, we find that first-row transition metals tend to yield insulating states, which is consistent with their spatially localized $3 d$ electrons and the relatively low symmetry of the $A_{\mathrm{p}}$ and $A_{\mathrm{t}}$ environments resulting from our structural relaxations. (Our relaxations are unconstrained symmetry wise, and guarantee the removal of electronic degeneracies in Jahn-Teller active species, if energetically favorable.) In contrast, we obtain metallic (Mo, Ru, Os) or marginally insulating $(\mathrm{Re})$ solutions when more extended $(4 d, 5 d)$ electrons are involved. (See Methods for a comment on these metallic states.) Thus, from a functional point of view, we reach a first relevant conclusion: It is possible to create metallic DWs in an insulating matrix by using $4 d$ and $5 d$ transition metals as dopants.

Let us stress that obtaining such highly conductive walls in a controllable manner is critical to some recent and exciting proposals of new electronics architectures based on ultra-dense inter-connected networks. Our results suggest that, by suitable doping, common ferroelastic perovskites may offer a convenient platform for such endeavors. As regards the nature of the conductivity in our walls, our results for the $4 d$ and $5 d$ dopants have traits (e.g., relatively small local magnetic moments) of a significant hybridization between the $d$ orbitals of the metal and the surrounding oxygen- $2 p$ orbitals, which suggests dispersive conductive bands and regular metallic behavior (i.e., band conductivity as opposed to activated hopping). However, a more detailed investigation of the electronic structure of our metallic DWs (which entails a considerable computational cost) would be required to elucidate this issue.

Figure 2 also reflects the fact that most of our doped DWs are magnetic. Indeed, we find both parallel (ferromagnetic or FM, e.g.,

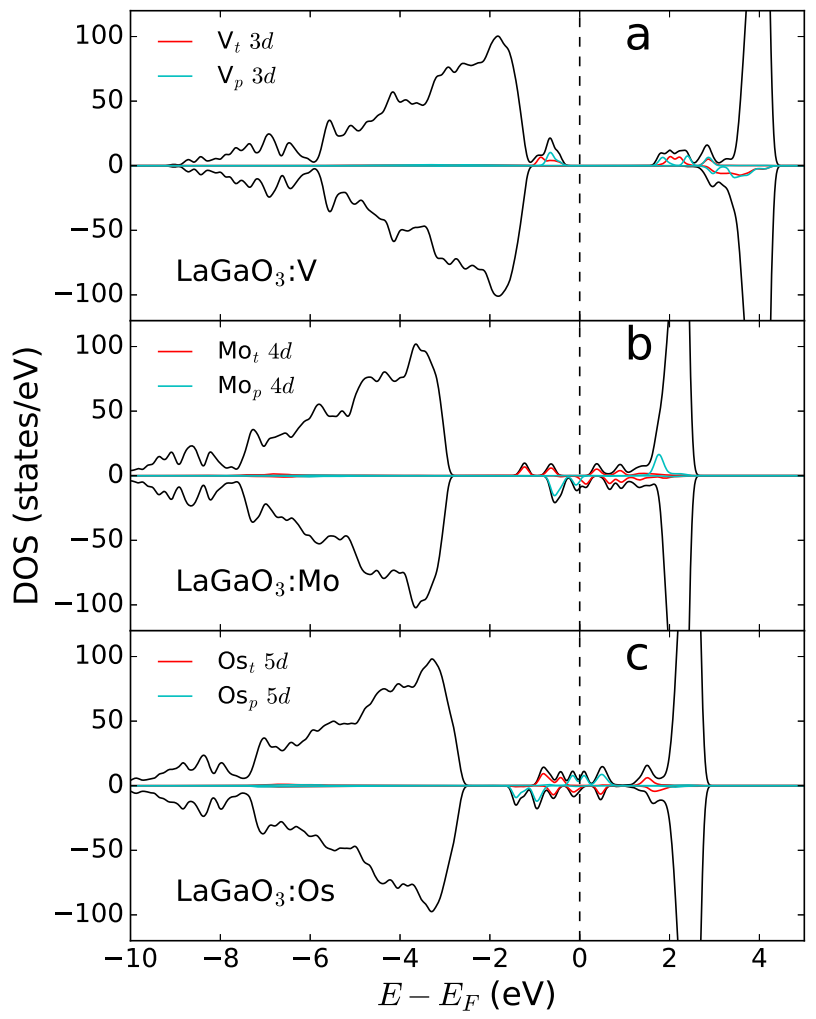

Fig. 3 Electronic structure of doped walls. Computed density of states (DOS) of $\mathrm{LaGaO}_{3}$ with domain walls doped with $\mathrm{V}$ (panel a), Mo (panel b), and Os (panel c). We give the total DOS (black line), dominated by the contribution of the domains, as well as the partial DOS corresponding to the dopants at the walls (red and cyan lines for dopants at the $A_{\mathrm{t}}$ and $A_{\mathrm{p}}$ sites, respectively)

for $\mathrm{V}$ and $\mathrm{Cr}$ ) and anti-parallel (ferrimagnetic or FiM, e.g., for Co and Mo) arrangements of the dopants' spins. Note that the DW dopants have very peculiar oxygen-coordination environments that differ from what is typical in perovskites and, more generally, in oxides. (For example, the planar $A_{p} \mathrm{O}_{4}$ structures of Fig. $1 \mathrm{c}$ are unheard of for most transition metals.) A detailed analysis of the mechanisms favoring particular magnetic interactions for particular dopants would be far from trivial, and falls beyond the scope of this work. Here we merely mention some remarkable aspects of our results. For example, we find some FiM metallic solutions (Mo, $\mathrm{Ru})$ as well as some FM insulating ones $(\mathrm{V}, \mathrm{Cr})$. This correlation is unusual among perovskite oxides, as super-exchange interactions favoring anti-parallel (FiM) spin alignments are typically dominant in insulators, while mechanisms favoring parallel (FM) spins tend to require (and dominate when we have) a metallic character. Further, our quantitative results suggest considerable effective exchange constants $J_{\text {eff }}$ in some cases (e.g., for the FM order in Vdoped walls, or for the FiM order in Co-doped walls), indicating relatively strong magnetic couplings. (See Supplementary Note 1 for an analysis of the magnetic interactions in the case of $\mathrm{Cr}$ doped $\mathrm{LaGaO}_{3}$, and a justification of the fact that in Fig. 2 we report a single effective coupling $J_{\text {eff }}$ between first-neighboring spins.) Hence, from a functional point of view, we have a second relevant conclusion: It is possible to create magnetic DWs in a non-magnetic matrix, including ferromagnetic insulating (and ferrimagnetic metallic) order, and featuring relatively strong magnetic interactions.

In addition, let us highlight a sticking structural and functional aspect. In some of our DWs, there is a clear off-centering of the doping cations, yielding a distortion that is analogous to those responsible for ferroelectricity in perovskite oxides like $\mathrm{BaTiO}_{3}$ 


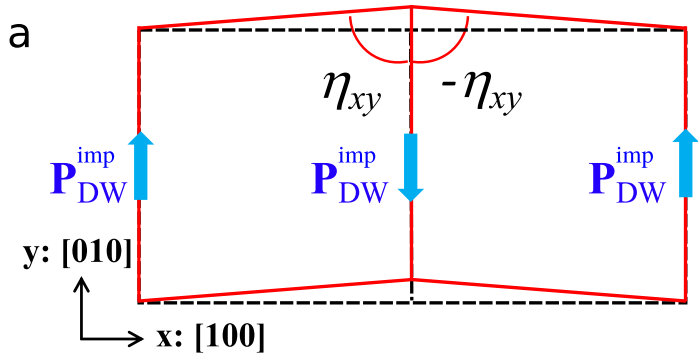

b $\quad \mathrm{V} @ \mathrm{LaGaO}_{3}$

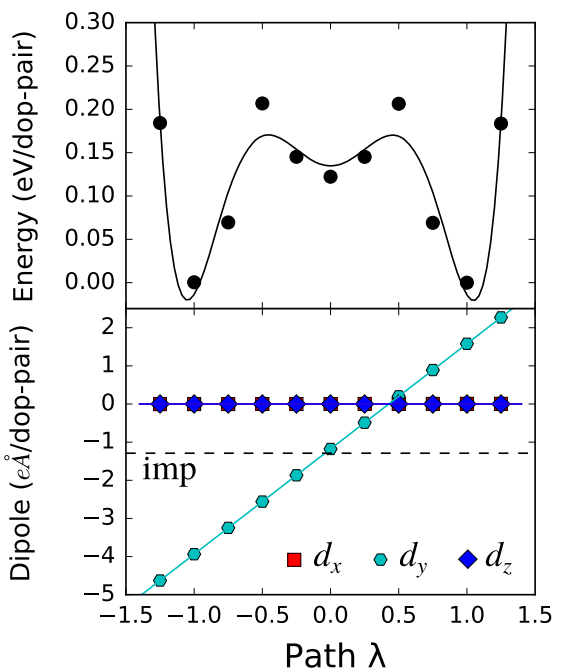

C $\quad \mathrm{Ti} @ \mathrm{LaGaO}_{3}$

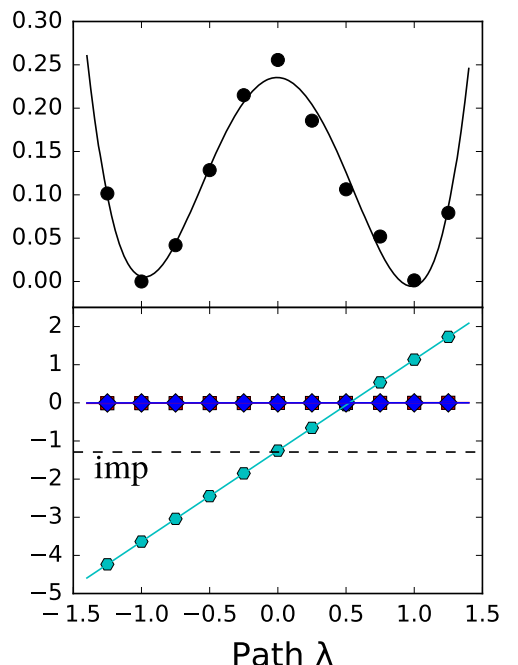

Fig. 4 Ferroelectric switching at doped walls. a Sketch of the symmetry breaking at our ferroelastic walls (the shear strain $\eta_{x y}$ changes sign from domain to domain), emphasizing that they present an improper polarization $P_{\mathrm{DW}}^{\mathrm{imp}}$ by construction. Note that the polarization of a wall cancels out with that of the next wall, so that the macroscopic dipole is null. $\mathbf{b}$ Computed energy and electric dipole $\left(d_{a}\right.$, where $a=x, y, z$ are the pseudo-cubic directions) as a function of the off-centering of the $\mathrm{V}$ cations at the $\mathrm{DWs}$ of $\mathrm{LaGaO}_{3}$ (see text for details). The variable $\lambda$ gives the position along a path that connects the two essentially equivalent polar states, located, respectively, at $\lambda=-1$ and $\lambda=1$. In the case of the energy, the points represent the first-principles data, and the line is the result of fitting these data so a 6th-order Landau-like polynomial function. c Same results for the Ti dopant, using a 4th-order polynomial in this case. Energies and dipoles are normalized per $A_{\mathrm{t}}-A_{\mathrm{p}}$ dopant

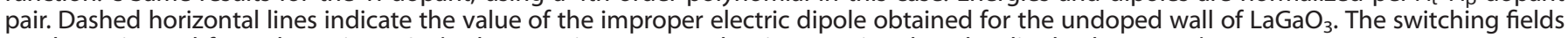
can be estimated from the ratio $\Delta E / \Delta d$, where $\Delta E$ is an energy barrier associated to the dipole change $\Delta d$

or $\mathrm{PbTiO}_{3}{ }^{21}$ The situation is best appreciated by comparing the Co-doped and V-doped walls shown in Fig. 2: in the former, both $\mathrm{Co}_{p}$ and $\mathrm{Co}_{t}$ cations are at high-symmetry positions (for example, $\mathrm{Co}_{\mathrm{p}}$ is at the center of an essentially planar $\mathrm{O}_{4}$ square); in contrast, in the latter the $\mathrm{V}$ cations move off-center, the situation being particularly clear for $\mathrm{V}_{\mathrm{p}}$.

The presence of a polar distortion at this kind of DW is not a surprise in itself: this is a ferroelastic boundary in which, by construction, inversion symmetry is broken and a polar distortion must exist, although it may be very small. (See sketch in Fig. 4a. For a theoretical discussion of ferroelastic walls in $\mathrm{CaTiO}_{3}$ and $\mathrm{SrTiO}_{3}$, see refs. ${ }^{22}$ and ${ }^{23}$, respectively.) Note that this polarity can be viewed as arising from flexoelectric couplings, i.e., the linear polar response to a strain gradient; indeed, because of the flexoelectric response, which is active in all materials, the finite strain discontinuity at a ferroelastic wall must necessarily produce a net electric polarization. In our case, an approximate estimate of the dipole moment associated to the $\mathrm{DWs}$ of pure $\mathrm{LaGaO}_{3}$ yields about $1.29 e \AA$ per group of four La cations, where $e$ is the elemental charge. (See Methods for the details of our polarization calculations.) Interestingly, this so-called improper polarization cannot be switched by application of an external electric field at the DW; rather, in order to switch such a DW polarization, we would need to act on the domains that give rise to the polar ferroelastic wall, and not on the DW itself.
However, in the V-doped DWs there is clearly an extra polar distortion. Here, we obtain a dipole moment of about -4 eA per group of four $A$ cations at the wall (equivalently per dopant pair). Further, by reversing the off-centering of the $V$ atoms, it is possible to identify the switched polarization state as a local minimum of the energy. Interestingly, for the reversed state we calculate about 1.5 eÅ; as shown in Fig. 4b, this means that the dipole change between these two states is $\sim 5.5 \mathrm{e} \AA$, with the mid-point between them $(\lambda=0$ in the figure) having an electric dipole of about -1.25 $e \AA$, which roughly coincides with the value of the built-in improper DW dipole. Similar results are obtained for other dopants, and in Fig. 4c we report the case of $\mathrm{Ti}$ as a second representative example. Then, we can compute the potential wells (double well for $\mathrm{Ti}$, triple well for $\mathrm{V}$ as shown in Fig. 4) that are characteristic of proper ferroelectricity, and even estimate the coercive fields necessary to make the DW polarization switch. (For example, following the procedure described in the caption of Fig. 4, we obtain about $11 \mathrm{MV} / \mathrm{cm}$ for Ti-doped walls.) We thus reach a new conclusion regarding DW engineering: By doping, it is possible to create ferroelectric DWs, with a sizeable and switchable polarization, within a paraelectric matrix.

While the above discussion is focused on the functional properties of the DWs of $\mathrm{LaGaO}_{3}$, we should note that we obtained qualitatively similar results for the doped $\mathrm{CaTiO}_{3}$ boundaries (see Supplementary Table 2 for a summary). However, 
as regards the magnitude of the effects observed, the doped walls of $\mathrm{LaGaO}_{3}$ yield generally stronger ones, which is the reason of our focus on this material. Note that there are several possible reasons for such quantitative differences (e.g., steric, as $\mathrm{La}^{3+}$ is larger than $\mathrm{Ca}^{2+}$, or electronic because of the different nominal valences); a detailed analysis remains for future work.

\section{DISCUSSION}

It is interesting to note that many of our doped DWs combine two or more of the properties mentioned above, and constitute genuine multi-functional objects. Most interestingly, our magnetic walls are always multiferroic. Indeed, because they are ferroelastic, they all present an improper polarization; thus, they are somewhat analogous to the so-called "Type II" multiferroics such as $\mathrm{TbMnO}_{3}{ }^{24}$ notwithstanding the fact that in such compounds the electric polarization is driven by the magnetization itself. In turn, the DWs presenting proper switchable ferroelectricity are genuine "Type I" multiferroics, as prototype material $\mathrm{BiFeO}_{3} .{ }^{25}$ Given the scarcity of such compounds, it is important to emphasize that it may be possible to obtain new magnetoelectric multiferroics, and even choose their character, by doping common ferroelastic perovskites.

It is also worth mentioning that, while in some cases we obtain relatively strong magnetic interactions at our doped walls, the existing information does not allow us to estimate the spinordering temperature in a reliable way. The main difficulty relates to the reduced dimensionality of our peculiar spin system, which poses the question of whether the Mermin-Wagner theorem, ${ }^{26}$ which states the absence of order in $1 D$ and $2 D$ spin systems governed by an isotropic Heisenberg model, applies or not in this case. To elucidate this issue, one needs to take into account the magnetic anisotropy associated to our DW spins. This would require an explicit relativistic calculation treating non-collinear magnetism; unfortunately, because of the large size of our simulations supercells, this is not feasible at the moment and, hence, an investigation of magnetic ordering at our DWs remains for the future.

While the focus of this work is on the effect of the dopants at the ferroelastic walls, we should point out that our first-principles results have interesting connections with (and may allow to benchmark) previous theories of this type of boundaries. Especially interesting are the works based on Ginzburg-Landau approaches, ${ }^{23,27-29}$ which discuss in detail the properties of the improper polarization naturally occurring at ferroelastic walls, and the couplings responsible for it. The phenomenological works also permit the investigation of situations that, because of the large supercells that would be required, are beyond the scope of current DFT simulations. One such example pertains to the behavior of the ferroelastic walls near the surface of the material, ${ }^{28,29}$ where a broadening and enhanced electric conductivity (which would be important in eventual applications of the DW functionality) is expected. First-principles simulations, which by default contain all the physical ingredients present in the phenomenological models, would be ideal to ratify or refine such interesting predictions. However, such large-scale DFT calculations are all but unfeasible today, and remain as a challenge for the future.

Our results suggest a few trends regarding the DW functionality that can be induced by different dopants, which can be used as guidelines for DW design. For example, we find that $3 d$ dopants tend to render insulating solutions, which $4 d$ and $5 d$ elements yield conductive walls. Also, we observe that smaller atoms ( $\mathrm{Ti}, \mathrm{V}$, $\mathrm{Cr}$ ) result in proper ferroelectric order at the DWs; in contrast, bigger ones remain in more symmetric configurations, so that the polarity of the corresponding (ferroelastic) walls is improper. Beyond these clear and physically sound trends, our results do not lend themselves to any simple interpretation. For example, as regards the computed magnetic interactions, we are currently unequipped to deduce general behaviors from our calculations, for two reasons; on one hand, we lack the basic theory (and ensuing expectations) to understand magnetic couplings between transition-metal atoms in such peculiar atomic environments; on the other hand, as stressed in the Methods section, the calculation of the magnetic couplings is most challenging for a first-principles scheme, and our quantitative results should be taken with caution (particularly for trouble atoms like V, Co, or Ni). Progress in this direction would probably require the use of more advanced and computationally costly first-principles methods, and is beyond the scope of the present work.

Finally, we should emphasize that the results discussed here are just a succinct summary of our findings and future possibilities. For example, among the investigated walls we also find cases in which proper ferroelectric distortions and metallicity occur simultaneously, thus yielding "polar metallic" behavior ${ }^{30,31}$ within a paraelectric insulating matrix. (See, e.g., the case of V-doped DWs in $\mathrm{CaTiO}_{3}$ in Supplementary Table 2). Further, many of the discussed DWs are candidates to present additional interesting properties (e.g., optical, magnetoresistive) and cross-coupling effects. Finally, as regards the materials in which these concepts might be realized in practice, let us stress that our results for $\mathrm{LaGaO}_{3}$ should be relevant to a large family of very common perovskites, such as $\mathrm{LaFeO}_{3}, \mathrm{LaCrO}_{3}$ and, more generally, all related orthorhombic compounds (ferrites, chromites, nickelates, manganites, titanates) with relatively large $A$ cations (e.g., La, $\mathrm{Pr}$, $\mathrm{Nd}, \mathrm{Sm}$ ). We thus believe that it will be experimentally feasible to pursue the striking effects described here, and even more exotic ones. For example, by working with orthorhombic nickelates, we may use a matrix that undergoes a metal-insulator transition, and may exploit non-trivial interactions between domains and walls. Hence, we hope our results will motivate efforts to implement this original strategy for creating functional nano-materials.

\section{METHODS}

For the first-principles calculations we use density functional theory (DFT) as implemented in the VASP code. ${ }^{32,33}$ The electronic wave functions are treated in a plane-wave basis cut off at $500 \mathrm{eV}$. The ionic cores are treated within the projector augmented wave (PAW) approximation, ${ }^{34}$ and we solve explicitly for the following electrons: $5 s, 5 p, 5 d$, and $6 s$ for La; $3 s, 3 p$, and $4 s$ for $\mathrm{Ca} ; 3 d, 4 s$, and $4 p$ for $\mathrm{Ga} ; 3 s, 3 p, 3 d$, and $4 s$ for $\mathrm{Ti} ; 3 s, 3 p, 3 d$, and $4 s$ for $\mathrm{V} ; 3 p, 3 d$, and $4 s$ for $\mathrm{Cr} ; 3 s, 3 p, 3 d$, and $4 s$ for $\mathrm{Mn} ; 3 p, 3 d$, and $4 s$ for Fe; $3 d$ and $4 s$ for $\mathrm{Co} ; 3 p, 3 d$, and $4 s$ for Ni; $4 p, 4 d$, and $5 s$ for Mo; $4 p, 4 d$, and $5 s$ for Ru; $5 p, 5 d$, and $6 s$ for Re; $5 p, 5 d$, and $6 s$ for Os; and $2 s$ and $2 p$ for $\mathrm{O}$. The so-called "PBEsol" (generalized gradient approximation revised for solids ${ }^{35}$ ) exchange-correlation functional is used.

In order to better treat the strong correlations typical of $3 d$ electrons, the so-called "Hubbard $U$ " (DFT $+U$ ) correction ${ }^{36}$ is adopted, using $U=3 \mathrm{eV}$ for all transition metals of the first row. In contrast, we do not use any such correction for $4 d$ (second row) and $5 d$ (third row) elements. These choices, particularly our using the same $U=3 \mathrm{eV}$ correction to all $3 d$ atoms, may seem questionable; yet, we think they are the best option in an exploratory investigation like the present one, for the following reasons. Note that using $U$ values between 2 and $4 \mathrm{eV}$ is a well-tested correction for studies of ferrites, chromites, and manganites (see refs. ${ }^{37-39}$ and references therein), and even for challenging cases like nickelates, as it has been found that $a$ small- $U$ correction successfully describes the magnetic and electronic properties of these compounds. ${ }^{40}$ Also, the weakly correlated nature of $4 d$ and $5 d$ cations has been shown in materials like $\mathrm{SrRuO}_{3}{ }^{41,42}$ and $\mathrm{LiOsO}_{3}{ }^{43}$ for which uncorrected DFT yields good agreement with experiments. It is nevertheless unclear whether we can apply to the present case the observations of previous DFT $+U$ studies of perovskite oxides, as the chemical environment of the cations in our DWs is very different from the case that is best studied, i.e., transition-metal atoms within octahedral $\mathrm{O}_{6}$ cages. Strictly speaking, if we wanted to run predictive DFT $+U$ simulations of our doped DWs, we would need an explicit calculation of the $U$ correction for each of the cases here considered. To further complicate things, note that the very use of the DFT $+U$ scheme would be questionable in some cases. Indeed, for species that are likely to present 
electronic states other than the high-spin configuration (e.g., $\mathrm{Co}^{3+}$ as well as the heavier $4 d$ and $5 d$ cations), one might in principle need different $U$ corrections for different spin states; but then, if we need to use casedependent exchange-correlation potentials, comparing energies is all but impossible. For all these reasons, resorting to a different simulation scheme, e.g., one based on hybrid functionals, ${ }^{35}$ would seem mandatory to obtain more reliable results. However, the plane-wave hybrid-functional implementations available to us involve a great computational burden, and are not ready to tackle the big simulation boxes required in our DW investigations. Hence, they are not an option today.

Thus, all things considered, we believe our blunt choice for the Hubbard$U$ correction is reasonable for an exploratory investigation like this one; we do not think there is a feasible and reliable way to do much better and, thus, we would rather avoid conveying the impression that we can fine tune our selections. Consequently, we must stress that some of the obtained results must be taken with a grain of salt, as we only expect them to be indicative of general trends. For example, the magnetic interactions are known to be very sensitive to the treatment of strong correlations, and we do not expect the particular values obtained to be very accurate. Also, as mentioned in the text, we obtain metallic (Mo, Ru, Os) or nearly metallic (Re) solutions for the DWs doped with $4 d$ and $5 d$ elements; yet, we should note that a more careful treatment of their electronic correlations might result in gapped solutions in some cases. Finally, following the usual practice in investigations of perovskite oxides, ${ }^{44-46}$ we do not use any $U$ correction for the empty $4 f$ electrons of La. Nevertheless, we explicitly checked that a correction of these levels has a negligible impact in the computed equilibrium structures.

A 160 -atom supercell (a $8 \times 2 \times 2$ multiple of the elemental perovskite unit, assuming a pseudo-cubic setting) is considered in most of our calculations, with a corresponding mesh of $1 \times 3 \times 3 \mathrm{k}$-points to sample the Brillouin zone. For each doping case, two magnetic structures are considered, i.e., with parallel and anti-parallel spin alignment between planar and tetrahedral doping sites. In order to get (local) energy minima, annealing calculations are run for each configuration; the resulting structures are then fully relaxed until the forces acting on each atom fall below $0.01 \mathrm{eV} / \AA . \AA$. These calculation conditions are on the verge of what we can tackle computationally, and yield quantitatively converged results for all the insulating DW states. Regarding the metallic cases, we can expect reliable results for the basic structural and electronic features; yet, the used $k$-point grid is probably not dense enough to get converged quantitative results for more challenging quantities, as for example the magnetic couplings.

We also run simulations of the undoped wall of $\mathrm{LaGaO}_{3}$ using a 320atom supercell (a $16 \times 16 \times 2$ mutiple of the elemental perovskite unit). By comparing the results obtained for 160- and 320-atom supercells, we conclude that the DW atomic and electronic structures are very well converged for the smaller simulation box. In contrast, the computed DW energy is not fully converged for the 160-atom cell: it varies from 74 to $87 \mathrm{~mJ} / \mathrm{m}^{2}$ when increasing the supercell size, suggesting that our DWs are still elastically coupled in the 160 -atom box. Note that this is not a concern in the context of the present work, which is focused on the local properties of doped DWs; yet, it may certainly warrant further consideration in the future.

The magnetic moments in Fig. 2 are obtained by projecting the occupied electronic wave functions onto atom-centered orbitals employed within the PAW scheme as implemented in VASP. This usually yields localized moments that are significantly below their nominal values, a result that can be partly attributed to a certain degree of hybridization (covalent bonding) with neighboring anions, and partly to the use of an incomplete local basis for the projections. Thus, our quantitative results for the magnetic moments have to be interpreted with caution; yet, they do reflect the occurrence of (dis)similar spin states between $A_{t}$ and $A_{\mathrm{p}}$ sites.

To compute electric dipoles we proceed in the following way. We consider a centro-symmetric configuration (typically, the cubic perovskite phase, which is assumed to have zero polarization) and calculate the distortion that connects it with the multi-domain configuration of interest. Then, by multiplying displacements of specific ions by their nominal ionic charge (e.g., +3 for La and Ga cations, -2 for oxygens, +3 for all our dopants), we obtain the corresponding local electric dipoles. Note that this approximation can be expected to yield approximate, but relatively small, dipole magnitudes. (In perovskite oxides, an accurate calculation of the atomic Born effective charges, using Berry's phase theory of polarization, ${ }^{47}$ usually yields values that exceed the nominal ones ${ }^{48}$.) Yet, our approach is particularly convenient when working with large supercells for which more accurate calculations would be computationally costly, which is why we adopt it here.

We used the matplotlib ${ }^{49}$ and VESTA ${ }^{50}$ softwares to prepare some of the figures.

\section{DATA AVAILABILITY}

The authors declare that the data supporting the present work are available from the corresponding author upon reasonable request.

\section{ACKNOWLEDGEMENTS}

Work funded by the Luxembourg National Research Fund through the CORE program (Grant FNR/C15/MS/10458889 NEWALLS). Computational resources provided by PRACE DECl-14 Grant 14DECI0042 "WALLS2CRYST".

\section{AUTHOR CONTRIBUTIONS}

Work executed by H.J.Z. and supervised by J.Í.

\section{ADDITIONAL INFORMATION}

Supplementary information accompanies the paper on the npj Computational Materials website (https://doi.org/10.1038/s41524-019-0229-5).

Competing interests: The authors declare no competing interests.

Publisher's note Springer Nature remains neutral with regard to jurisdictional claims in published maps and institutional affiliations.

\section{REFERENCES}

1. Rabe, K. M., Ahn, C. H. \& Triscone, J. (eds.) Physics of Ferroelectrics, A Modern Perspective (Springer Berlin Heidelberg, Berlin, Heidelberg, 2007).

2. Tagantsev, A., Cross, L. E. \& Fousek, J. Domains in Ferroic Crystals and Thin Films (Springer, 2010).

3. Salje, E. K. Multiferroic domain boundaries as active memory devices: trajectories towards domain boundary engineering. ChemPhysChem 11, 940-950 (2010).

4. Catalan, G., Seidel, J., Ramesh, R. \& Scott, J. F. Domain wall nanoelectronics. Rev. Mod. Phys. 84, 119-156 (2012).

5. McQuaid, R. G., Campbell, M. P., Whatmore, R. W., Kumar, A. \& Gregg, J. M. Injection and controlled motion of conducting domain walls in improper ferroelectric Cu-Cl boracite. Nat. Commun. 8, 15105 (2017).

6. Lu, H. et al. Mechanical writing of ferroelectric polarization. Science 336, 59-61 (2012).

7. Sharma, P. et al. Nonvolatile ferroelectric domain wall memory. Sci. Adv. 3, e1700512 (2017).

8. Aird, A. \& Salje, E. K. H. Sheet superconductivity in twin walls: experimental evidence of $\mathrm{WO}_{3-x}$. J. Phys.: Condens. Matter. 10, L377 (1998).

9. Seidel, J. et al. Conduction at domain walls in oxide multiferroics. Nat. Mater. 8 , 229-234 (2009).

10. Farokhipoor, S. \& Noheda, B. Conduction through $71^{\circ}$ domain walls in $\mathrm{BiFeO}_{3}$ thin films. Phys. Rev. Lett. 107, 127601 (2011).

11. Guyonnet, J., Gaponenko, I., Gariglio, S. \& Paruch, P. Conduction at domain walls in insulating $\mathrm{Pb}\left(\mathrm{Zr}_{0,2} \mathrm{Ti}_{0.8}\right) \mathrm{O}_{3}$ thin films. Adv. Mater. 23, 5377-5382 (2011).

12. Diéguez, O., Aguado-Puente, P., Junquera, J. \& Íñiguez, J. Domain walls in a perovskite oxide with two primary structural order parameters: first-principles study of $\mathrm{BiFeO}_{3}$. Phys. Rev. B 87, 024102 (2013).

13. Farokhipoor, S. et al. Artificial chemical and magnetic structure at the domain walls of an epitaxial oxide. Nature 515, 379-383 (2014).

14. Biškup, N. et al. Insulating ferromagnetic $\mathrm{LaCoO}_{3-\delta}$ films: a phase induced by ordering of oxygen vacancies. Phys. Rev. Lett. 112, 087202 (2014).

15. Becher, $C$. et al. Strain-induced coupling of electrical polarization and structural defects in $\mathrm{SrMnO}_{3}$ films. Nat. Nanotechnol. 10, 661-665 (2015).

16. Glazer, A. M. The classification of tilted octahedra in perovskites. Acta Crystallogr. Sect. B 28, 3384-3392 (1972).

17. Lufaso, M. W. \& Woodward, P. M. Prediction of the crystal structures of perovskites using the software program SPuDS. Acta Crystallogr. Sect. B 57, 725-738 (2001).

18. Chen, P. et al. Energetics of oxygen-octahedra rotations in perovskite oxides from first principles. Phys. Rev. B 97, 024113 (2018)

19. Wang, Y., Liu, X., Yao, G.-D., Liebermann, R. \& Dudley, M. High temperature transmission electron microscopy and $\mathrm{x}$-ray diffraction studies of twinning and the phase transition at $145^{\circ} \mathrm{C}$ in $\mathrm{LaGaO}_{3}$. Mater. Sci. Eng.: A 132, 13-21 (1991). 
20. Kennedy, B. J., Vogt, T., Martin, C. D., Parise, J. B. \& Hriljac, J. A. Pressure-induced orthorhombic to rhombohedral phase transition in $\mathrm{LaGaO}_{3}$. J. Phys.: Condens. Matter 13, L925 (2001).

21. King-Smith, R. D. \& Vanderbilt, D. First-principles investigation of ferroelectricity in perovskite compounds. Phys. Rev. B 49, 5828 (1994).

22. Barone, P., Di Sante, D. \& Picozzi, S. Improper origin of polar displacements at $\mathrm{CaTiO}_{3}$ and $\mathrm{CaMnO}_{3}$ twin walls. Phys. Rev. B 89, 144104 (2014).

23. Schiaffino, A. \& Stengel, M. Macroscopic polarization from antiferrodistortive cycloids in ferroelastic $\mathrm{SrTiO}_{3}$. Phys. Rev. Lett. 119, 137601 (2017).

24. Khomskii, D. Classifying multiferroics: mechanisms and effects. Physics 2, 20 (2009).

25. Catalan, G. \& Scott, J. F. Physics and applications of bismuth ferrite. Adv. Mater. 21, 2463-2485 (2009).

26. Mermin, N. D. \& Wagner, H. Absence of ferromagnetism or antiferromagnetism in one- or two-dimensional isotropic heisenberg models. Phys. Rev. Lett. 17, 1133-1136 (1966).

27. Morozovska, A. N., Eliseev, E. A., Glinchuk, M. D., Chen, L.-Q. \& Gopalan, V. Interfacial polarization and pyroelectricity in antiferrodistortive structures induced by a flexoelectric effect and rotostriction. Phys. Rev. B 85, 094107 (2012).

28. Morozovska, A. N., Eliseev, E. A., Kalinin, S. V., Qing Chen, L. \& Gopalan, V. Surface polar states and pyroelectricity in ferroelastics induced by flexo-roto field. Appl. Phys. Lett. 100, 142902 (2012).

29. Eliseev, E. A. et al. Conductivity of twin-domain-wall/surface junctions in ferroelastics: Interplay of deformation potential, octahedral rotations, improper ferroelectricity, and flexoelectric coupling. Phys. Rev. B 86, 085416 (2012).

30. Benedek, N. A. \& Birol, T. "Ferroelectric" metals reexamined: fundamental mechanisms and design considerations for new materials. J. Mater. Chem. C. 4, 4000-4015 (2016).

31. Zhao, H. J. et al. Meta-screening and permanence of polar distortion in metallized ferroelectrics. Phys. Rev. B 97, 054107 (2018).

32. Kresse, G. \& Furthmüller, J. Efficient iterative schemes for ab initio total-energy calculations using a plane-wave basis set. Phys. Rev. B 54, 11169-11186 (1996).

33. Kresse, G. \& Joubert, D. From ultrasoft pseudopotentials to the projector augmented-wave method. Phys. Rev. B 59, 1758-1775 (1999).

34. Blöchl, P. E. Projector augmented-wave method. Phys. Rev. B 50, 17953-17979 (1994).

35. Perdew, J. et al. Restoring the density-gradient expansion for exchange in solids and surfaces. Phys. Rev. Lett. 100, 136406 (2008).

36. Dudarev, S. L., Botton, G. A., Savrasov, S. Y., Humphreys, C. J. \& Sutton, A. P. Electron-energy-loss spectra and the structural stability of nickel oxide: an LSDA + U study. Phys. Rev. B 57, 1505-1509 (1998).

37. Hong, J., Stroppa, A., Íñiguez, J., Picozzi, S. \& Vanderbilt, D. Spin-phonon coupling effects in transition-metal perovskites: A DFT $+U$ and hybrid-functional study. Phys. Rev. B 85, 054417 (2012).
38. Zhao, H. J., Íñiguez, J., Chen, X. M. \& Bellaiche, L. Origin of the magnetization and compensation temperature in rare-earth orthoferrites and orthochromates. Phys. Rev. B 93, 014417 (2016).

39. Gibert, M., Zubko, P., Scherwitzl, R., Íñiguez, J. \& Triscone, J. M. Exchange bias in $\mathrm{LaNiO}_{3}-\mathrm{LaMnO}_{3}$ superlattices. Nat. Mater. 11, 195-198 (2012).

40. Catalano, S. et al. Rare-earth nickelates $\mathrm{RNiO}_{3}$ : thin films and heterostructures. Rep. Prog. Phys. 81, 046501 (2018).

41. Etz, C. et al. Indications of weak electronic correlations in $\mathrm{SrRuO}_{3}$ from firstprinciples calculations. Phys. Rev. B 86, 064441 (2012).

42. Miao, N., Bristowe, N. C., Xu, B., Verstraete, M. J. \& Ghosez, P. First-principles study of the lattice dynamical properties of strontium ruthenate. J. Phys.: Condens. Matter 26, 035401 (2013).

43. Xiang, H. J. Origin of polar distortion in $\mathrm{LiNbO}_{3}$-type "ferroelectric" metals: role of A-site instability and short-range interactions. Phys. Rev. B 90, 094108 (2014).

44. May, S. J. et al. Quantifying octahedral rotations in strained perovskite oxide films. Phys. Rev. B 82, 014110 (2010).

45. González-Vázquez, O. E., Wojdeł, J. C., Diéguez, O. \& Íñiguez, J. First-principles investigation of the structural phases and enhanced response properties of the $\mathrm{BiFeO}_{3}-\mathrm{LaFeO}_{3}$ multiferroic solid solution. Phys. Rev. B 85, 064119 (2012).

46. Hou, Y. S., Xiang, H. J. \& Gong, X. G. Intrinsic insulating ferromagnetism in manganese oxide thin films. Phys. Rev. B 89, 064415 (2014).

47. King-Smith, R. D. \& Vanderbilt, D. Theory of polarization of crystalline solids. Phys. Rev. B 47, 1651-1654 (1993).

48. Zhong, W., King-Smith, R. D. \& Vanderbilt, D. Giant LO-TO splittings in perovskite ferroelectrics. Phys. Rev. Lett. 72, 3618 (1994).

49. Hunter, J. D. Matplotlib: a 2d graphics environment. Comput. Sci. Eng. 9, 90-95 (2007).

50. Momma, K. \& Izumi, F. Vesta 3 for three-dimensional visualization of crystal volumetric and morphology data. J. Appl. Crystallogr. 44, 1272-1276 (2011).

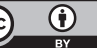

Open Access This article is licensed under a Creative Commons Attribution 4.0 International License, which permits use, sharing, adaptation, distribution and reproduction in any medium or format, as long as you give appropriate credit to the original author(s) and the source, provide a link to the Creative Commons license, and indicate if changes were made. The images or other third party material in this article are included in the article's Creative Commons license, unless indicated otherwise in a credit line to the material. If material is not included in the article's Creative Commons license and your intended use is not permitted by statutory regulation or exceeds the permitted use, you will need to obtain permission directly from the copyright holder. To view a copy of this license, visit http://creativecommons. org/licenses/by/4.0/.

(c) The Author(s) 2019 